\title{
IV. Weltwirtschaftskrise und Arbeitsmarktkrise (1929-1932)
}

Die Bestandsaufnahme der Berliner Gesandtschaft und der Konsulate im Frühjahr 1930 zeichnete ein düsteres Bild der Arbeitsbedingungen der Tschechoslowaken im Deutschland der Weltwirtschaftskrise. Im allgemeinen erhielten die Altansässigen, wenn auch nicht immer ohne bürokratische Hürden, den Befreiungsschein; den nach dem 30. April 1923 Zugewanderten hingegen wurden Arbeits- und Aufenthaltserlaubnis nun grundsätzlich verweigert. Behandelte man Tschechoslowaken deutscher Nationalität auch etwas freundlicher als Nationaltschechen, so kritisierte doch auf einer vom Sudetendeutschen Heimatbund (SHB) in Dresden einberufenen, in der Mehrzahl von Staatsbürgern der ČSR besuchten Protestversammlung der Deutschen der ehemaligen Donaumonarchie am 10. April 1930 der sächsische SHB-Vorsitzende Grimme, im Zuge der krisenbedingten Entlassungen werde der Unterschied zwischen Tschechen und tschechoslowakischen Bürgern deutscher Nationalität ignoriert, obwohl letztere sich als „Kämpfer für Deutschland“ fühlten. Laut Bericht des Chemnitzer Konsulats band etwa das Landesarbeitsamt Erfurt die Arbeitserlaubnis an einen „Deutschstämmigkeitsnachweis". Auswärtiges Amt und Arbeitsverwaltung werteten dies nicht als Vertragsverstoß, weil eine positive Diskriminierung vorliege; das Recht auf wohlwollende Behandlung von „ausländischen Stammeszugehörigen“ werde „von allen Ländern als ein Hoheitsrecht in Anspruch genommen".

Die Wende zur konsequenten Ablehnungspraxis, hinsichtlich derer die Behörden an einem Strang zogen, hatte das Dresdner Konsulat schon im Herbst 1929 eintreten sehen. Die dortige Kreishauptmannschaft entziehe Empfängern von Arbeitslosenunterstützung die Aufenthaltsbewilligung und weise seit langem ansässige Kräfte aus, sobald sie der Fürsorge zur Last fielen. Das Chemnitzer Konsulat berichtete von außergesetzlichem Druck auf die Arbeitgeber, Tschechoslowaken zu entlassen. Die Mittel des Prager Fürsorgeressorts zur Unterstützung der Landsleute in Not waren höchst unzureichend; um eine zusätzliche Belastung des heimischen Arbeits- und des Wohnungsmarktes durch Repatriierungen zu verhindern, bemühte sich das Ministerium um einen außerordentlichen Kredit in Höhe von 2 Millionen Kronen. Bis Anfang 1932 hatte sich die Lage verschärft, der Nothilfeetat in Höhe von 480000 Kronen war nun eklatant zu knapp. ${ }^{1}$

Ungeachtet der Krise und der reichsdeutschen Pressionen warben Stimmen von Gewicht nach wie vor für Freizügigkeit. Mitte Februar 1930 reichte die Konföderation der

1 AKPR, T 12/25, Karton 135, C V, MZV an KPR, 12.5.1930. - SÚA, MSP, Karton 1808, E 4/b-23/1930, MSP an MZV, 22. 4.1930. - Tschechoslowakisches Konsulat Köln an Tschechoslowakische Gesandtschaft Berlin, 23.4.1930. - Konsulat Bremen, 26. 4. 1930. - Generalkonsulat München, 19. 5. 1930. - Generalkonsulat Dresden, 21.5. 1930. - Konsulat Chemnitz, 12.6. 1930. - SÚA, MSP, Karton 2946, E 4221/1930-32, Tschechoslowakisches Generalkonsulat Dresden an Tschechoslowakische Gesandtschaft Berlin, 26.11.1930. - AKPR, D 8692, Karton 120, MSP an PMR, 22.10.1930 bzw. 23.1.1932. - PA, R 73 839, AA an RAM, 29.10.1931. - Präsident der Reichsanstalt für Arbeitsvermittlung und Arbeitslosenversicherung an RAM, 7.12.1931. $\mathrm{RAM}$ an AA, 28.12.1931. 
Arbeitgeberverbände ein ceterum censeo zur Stornierung des Arbeitsmarktgesetzes $e^{2}{ }^{2}$; der Mährische Industriellenverband wollte zumindest längerfristig durch Gegenseitigkeitsverträge die Freizügigkeit auf den Arbeitsmärkten wiederhergestellt sehen. ${ }^{3}$ Angesichts der Vertragsverletzungen vor allem Sachsens faßte andererseits das Außenministerium im Dezember 1930 eine Démarche ins Auge ${ }^{4}$; das Fürsorgeministerium wollte die Zügel anziehen. ${ }^{5}$ Der Kongreß der Auslandstschechen und -slowaken befürwortete im Prinzip zwar Arbeitsmarktfreiheit, andererseits aber auch Retorsionen gegen Unfreundlichkeiten anderer Staaten. Die ČSR solle beim Internationalen Arbeitsamt auf die Gleichstellung von In- und Ausländern dringen und sich mit allen Staaten, die in ihren Grenzen eine größere Anzahl tschechoslowakischer Bürger beherbergten, in diesem Sinne vereinbaren. ${ }^{6}$

Auch unabhängig von der reichsdeutschen Praxis wurde nun eine Reservierung qualifizierter Arbeitsplätze für Inländer verfochten. Im November 1929 riefen mehrere Vereinigungen von Studenten technischer Fächer nach einem Verbot der Ausländerbeschäftigung, wo diese einheimischen Ingenieuren zum Schaden gereiche ${ }^{7}$; der Verband der Absolventen der Prager Handelshochschule wollte Anfang 1931 ins Bewilligungsverfahren eingeschaltet werden. ${ }^{8}$ Der Reichenberger Allgemeine Angestellten-Verband suchte angesichts der "gerade in unseren hauptsächlichen Organisationsgebieten" bedeutenden „Stellenlosigkeit“ unter den arbeitslosen Mitgliedern nach „Ersazz für den einen oder anderen ausländischen Spezialisten ${ }^{\text {" }}$, die Gesamtgewerkschaftliche Vereinigung der tschechoslowakischen Privatbeamten, Werkmeister und Angestellten forderte den Unternehmen den Nachweis ab, sich bei Stellenbesetzungen zunächst um Inländer bemüht zu haben. ${ }^{10}$ Solche Interessenpolitik stieß allerdings an sachbedingte Grenzen: Sogar der Reichenberger Allgemeine Angestellten-Verband gutachtete trotz 3000 arbeitsloser Mitglieder meist positiv, weil „besondere Fachkräfte mit derartiger fachlicher Qualifikation" gesucht würden, so daß „eine Zuweisung aus dem Stande der stellenlosen Mitglieder nicht möglich" sei. ${ }^{11}$

Richtete sich der Protektionismus, soweit krisenbedingt, gegen Ausländer generell, so kam im Fall der Deutschen zusätzlich die Nationalpolitik ins Spiel. Von der Presse kolportierte Gerüchte, eine Zählung aller ausländischen Arbeitnehmer werde vorbereitet,

2 SÚA, MSP, Karton 1807, E 4a/1930, Konföderation der tschechoslowakischen Arbeitgeberverbände an MSP, 11.2. 1930.

3 BVMI 1930, S. 127 f. - Ähnlich der Tenor in: MDHI 11 (1930), S. 67.

4 SÚA, MSP, Karton 2946, E 4221/1930-32, MZV an MSP, 10.12.1930.

5 SÚA, MSP, Karton 1808, E 4/b-23/1931, Aktenvermerk MSP vom 17.11. 1930.

6 HŘ vom 3. 9. 1932.

7 SÚA, MSP, Karton 1807, E 4/a/1930, MŠaNO an MSP, 3.4. 1930. - Aktiv geworden waren der Verein der Studenten des Maschinenbau- und des elektrotechnischen Ingenieurwesens sowie der Verein der Ingenieursstudenten.

8 SÚA, MSP, Karton 1807, E 4/a/1931, Verein der Hörer der Handelsingenieurs-wesens (!) an MSP, 10.2.1931.

9 SÚA, MSP, Karton 1807, E 4/a, 1932, Allgemeiner Angestellten-Verband (Reichenberg) an MSP, 3. 3. und 30.5.1932.

10 SÚA, MSP, Karton 1807, E 4/a, 1932, „Jednota“ - Gesamtgewerkschaftliche Vereinigung der tschechoslowakischen Privatbeamten, Werkmeister und Angestellten an MSP, 6. 4. 1932.

11 SÚA, MSP, Karton 1807, E 4/a, 1932, Aktenvermerk MSP vom 26. 9. 1932. - Allgemeiner Angestellten-Verband (Reichenberg) an MSP, 29. 10. 1932. 
erwiesen sich zwar als substanzlos ${ }^{12}$; trotzdem wehte nun ein kühlerer Wind aus der nationaltschechischen Ecke. Der DHI, der in der Arbeitsmarktfrage das gesamte Unternehmerlager hinter sich wußte und sich auch der Zustimmung aller maßgebenden Gewerkschaften versichert hatte, witterte nicht zu Unrecht die Urheberschaft für eine in der zweiten Jahreshälfte 1930 zu beobachtende deutliche Verschärfung der Gangart der Behörden bei den Volkstumsverbänden. ${ }^{13}$ Der „Venkov“ vom 26. Februar 1931 kritisierte die Zahl der im ersten Quartal dieses Jahres neu erteilten bzw. verlängerten Arbeitserlaubnisse als untragbar hoch ${ }^{14}$, der Nordböhmische Volkstumsverein und verschiedene Berufsverbände forderten Entlassungen und die Verweigerung von Aufenthaltsverlängerungen, der „Hraničáŕ“ rief allgemein nach Hilfe für die Tschechen im Grenzland. ${ }^{15}$ Von krisenbedingten Lohnsenkungen, widergesetzlichen Arbeitszeitverlängerungen und Kündigungen sah im März 1931 die Gewerkschaftszentrale der tschechoslowakischen Beamten- und Angestelltenorganisationen die Tschechen vergleichsweise härter als die Deutschen betroffen. Diese - so hieß es - lehnten zusammen mit dem tschechoslowakischen Staat auch den Staatsdienst ab; Domäne der Deutschen sei die Privatwirtschaft, wo sie aufgrund ihrer besseren Ausbildung und der positiven Voreingenommenheit deutscher Arbeitgeber früher geheuert und später gefeuert würden. Aus diesem Grund forderte die Zentrale die Vergabe höherer Angestelltenpositionen auf der Grundlage eines Nationalitätenschlüssels. ${ }^{16}$ Am 15. Mai 1932 applaudierte der "Venkov" einer Ankündigung, das Fürsorgeministerium bereite unter dem Eindruck von Presseprotesten die Überprüfung der Bewilligungen in verschiedenen Branchen vor, um sie mit dem Arbeitsmarktgesetz in Einklang zu bringen. Die Hoffnung war, vielen Reichsdeutschen und Österreichern werde gekündigt. ${ }^{17}$ Daß die Existenz solcher Aktivitäten offiziell dementiert worden war, stellte für die Reichsdeutschenvereine geradezu ein Beleg für die Richtigkeit der Information dar. Zwar würden von „nationaltschechischer Seite die Prager Regierungsstellen nicht mehr als ihr willfähriges Instrument angesehen“, da diese derzeit "keinerlei Interesse an einer Verschärfung der Angelegenheit" hätten; daß führende Kreise in Wirtschaft und Politik sich nun „der Angelegenheit auf kaltem Wege" bemächtigen wollten und auf dem Weg über die Verwaltungsräte oder durch andere Hebel, etwa Devisenzuteilungen, Druck ausübten, sei der gefährlichere Weg. ${ }^{18}$

Verschärfend wirkte die Furcht vor den Deutschen als Sicherheitsrisiko. Im Juni 1931 versandte die Arbeitsgemeinschaft der Auslandsingenieure im Verein Deutscher Ingenieure (VDI) an ihre Mitglieder einen Fragebogen, der als Aufruf zur Industriespionage mißverstanden werden konnte - und, wie das Auswärtige Amt beobachtete, tatsächlich

12 Deutsche Allgemeine Zeitung vom 18.12.1930. - MDHI 11 (1930), S. 906.

13 DHI, Tätigkeitsbericht 1930, S. 35.

14 Venkov vom 26.2. 1931. - PT vom 27.2. 1931.

15 SÚA, MSP, Karton 1810, E 4/a/1931, Nordböhmischer Volkstumsverein an MV, 16.3.1931. Zentralverein der Werkmeister und Beamten der Metallindustrie an MSP, 30.12.1931. - HR vom 28. 11. 1931.

16 SÚA, MSP, Karton 1807, E 4/a/1931, Gewerkschaftszentrale der tschechoslowakischen Beamten- und Angestelltenorganisationen an MSP, 17.3.1931.

17 Venkov vom 15. 5. 1932.

18 BayHStA, MA 103 108, Pflock an BayStMÄWA, 23. 5. 1932. - Von diesem mit der Bitte um Vorstellung beim AA weitergegeben: Vgl. BayStMÄWA an Bayerische Gesandtschaft Berlin, 8. 6. 1932 (Konzept). 
"die befürchtete Beurteilung" fand. Die „Československá obrana“ hetze, so der VDI, unter der Überschrift „Berlin lädt seine deutschen Kollegen in der ČSR zur Spionage ein“ „in der übelsten Weise gegen alles Deutsche“. Daß auch die offiziöse „Prager Presse" vom 24. September die Absicht zur "Schaffung einer weitreichenden Wirtschafts-Spionage-Organisation" unterstellte, machte nach Auffassung der Gesandtschaft den ohnehin schweren Stand der Reichsdeutschen noch komplizierter. ${ }^{19}$ Das Dementi des VDI wurde von der "Prager Presse“ als "Lippenbekenntnisse" eingestuft. ${ }^{20} \mathrm{Am} 4$. Oktober forderte der „Venkov“, alle Reichsdeutschen in der Industrie des Landes seien zu observieren; die Inflation deutscher Ingenieure in der Schwerindustrie und der Chemie, Folge der bedeutenden Position deutscher Kapitaleigner dort, gebiete, „die Personalverhältnisse unserer Industrieunternehmungen peinlichst zu beachten ". ${ }^{21}$ Angesichts wachsender innenpolitischer Spannungen und einer Gefährdung der Staatssicherheit durch „Hakenkreuzler“ und Beschäftigungskrise forderten Volkstumsvereine und Nationalrat die Reparatur der bei der Nostrifizierung von Unternehmen gemachten Fehler. ${ }^{22}$

Die Behörden blieben von solchen Stimmungen und Einflüssen nicht unberührt: $\mathrm{Da}$ bei der Besetzung von Spitzenpositionen der Wirtschaft die Arbeitsämter im Regelfall nicht mitwirkten, gedachte die nordböhmische Landeszentrale für Arbeit, sich durch amtliche Ausschreibung in solche Vorgänge einzuschalten. Sie hatte auch eine Statistik der an Ausländer erteilten Bewilligungen erstellt; geplant war, die Arbeitgeber auszuforschen, ob und wie diese Kräfte ersetzt werden könnten. ${ }^{23}$ Für solche Verhärtungen war Deutschland Anlaß oder zumindest Vorwand: Unter Berufung auf das Vorbild des Nachbarn erwog Anfang 1932 ein nordböhmisches Arbeitsamt die Entlassung aller Ausländer ohne Rücksicht auf Rechtsansprüche. Unterstützung erfuhr dieses Projekt von der nordböhmischen Landeszentrale: Die Arbeitslosigkeit im Grenzgebiet sei ja schließlich wesentlich der Entlassung früher in Deutschland beschäftigter Arbeitskräfte geschuldet. ${ }^{24}$

Waren dies nur Forderungen und Absichten, so bewegte sich die Verwaltungspraxis nun doch in eine Richtung, die nicht mehr ohne weiteres mit dem Geist, ja nicht einmal mit dem Buchstaben des Rechts und auch nicht mit dem bilateralen Vertrag in Einklang zu bringen war. Angesichts sich häufender Berichte über die schematische Ablehnung von Weiterbeschäftigungsanträgen durch die Behörden „mittels vorgedruckter Formulare" riet die Arbeitgeberhauptstelle des DHI, Unternehmen sollten sich auf die zwischenstaatlich vereinbarte Härtefallklausel berufen, den Rekurs und seine aufschiebende Wirkung nutzen und Ablehnungen an den Verband weiterleiten. ${ }^{25}$ Dieser sollte auch

19 PA, R 89 715, VDI an AA, 19.9.1931. - PA, R 89 761, AA an Gesandtschaft Prag, 30. 9. 1931. PA, R 89715 , Gesandtschaft Prag an AA, 1.10.1931.

20 Die Gegendarstellung des VDI im Detail und die Antwort hierauf in der PP vom 11. 10. 1931.

21 Venkov vom 4. 10.1931. - Vgl. auch PA, R 89 715, Gesandtschaft Prag an AA, 7. 10. 1931.

22 Č 12 (1932), Nr. 3, S. 75 und Nr. 4, S. 107 f. - Fast gleichlautend die Verlautbarung des Nationalrats: Vgl. SÚA, NRČ, P, Karton 138/1/98, Exposé für den Abgeordneten Antonín Hajn zur Sitzung des Exekutivausschusses des Tschechoslowakischen Nationalrats am 7.4.1932.

23 SÚA, MSP, Karton 1807, E 4/a/193O, Landesinspektorat der Bezirksarbeitsäm-ter/Landeszentrale für Arbeit für Nordböhmen in Reichenberg an MSP, 22. 12. 1930.

24 SÚA, MSP, Karton 1807, E 4/a, 1932, Landesinspektorat der Bezirksarbeitsämter in Reichenberg/Landeszentrale für Arbeit für Nordböhmen an MSP, 5. 1.1932.

25 MDHI 12 (1931), S. 25 f. 
eingeschaltet werden, wo Bezirks- und Polizeibehörden in der Tagespresse veröffentlichte, nach Auffassung der Arbeitgeberhauptstelle widergesetzliche „Kundmachungen über die Handhabung " des Gesetzes anwandten. ${ }^{26}$

Die im Herbst 1931 geäußerte Befürchtung der Reichsdeutschenvereine, eine rigidere Auslegung der bilateralen Vereinbarung durch das deutsche Reich werde sich in der ČSR unter dem Druck der Presse unverzüglich auswirken, bewahrheitete sich: Verschleppungen und Versagungen von Verlängerungen wurden registriert, Rekurse waren zum Teil seit Monaten anhängig. ${ }^{27}$ Am 9. Januar 1932 protestierte die deutsche Gesandtschaft gegen die Praxis, nach sechsjähriger Aufenthaltsdauer die Bewilligung zu entziehen - „eine ganz besondere Härte“, die den Eindruck vermittle, „gewisse nachgeordnete tschechoslowakische Behörden wollten verhindern, daß reichsdeutsche Arbeitnehmer in den Genuß der Vergünstigung des Artikels 2 der genannten Vereinbarung gelangen “ ${ }^{28}$ Ende Mai 1932 übergab die Gesandtschaft in Prag eine lange Liste von Problemfällen. ${ }^{29}$ Beschäftigungsbewilligungen, so auch die Kritik des Hauptverbandes an den ebenso willkürlichen wie teilweise unsinnigen administrativen Praktiken, könnten unmöglich an nicht näher spezifizierte Bedingungen geknüpft werden. Die Usance, für jeden Ausländer die Einstellung eines Einheimischen zur Auflage zu machen, sei zumindest bei leitenden und Vertrauensposten weltfremd. Amtliche Verfügungen, die Erlaubnis werde hinfällig, falls nicht binnen zwei Monaten eine - nicht näher definierte - „Vorkehrung“ zur Einarbeitung eines Inländers getroffen werde, sprächen jeglicher Rechtssicherheit Hohn. Direktoren großer Unternehmen könnten eben nicht angelernt werden; niemandem sei zuzumuten, einen Nachfolger, der ihn dann verdränge, hochzuziehen. Weltfern und wirtschaftsfremd sei auch die Klausel, die Zahl der einheimischen Beschäftigten eines Unternehmens dürfe nicht reduziert werden. Die Tendenz, die Laufzeit der Bewilligungen auf sechs Monate zu halbieren, zeitige lediglich administrativen und finanziellen Mehraufwand. ${ }^{30}$

Der „Wirtschaft“ zufolge verfuhren nun Gerichte sogar bei der Eintragung von Prokuristen ins Handelsregister nach dem Arbeitsmarktgesetz - eine Praxis, die einige Male vom Obergericht in Prag, in einem Fall sogar vom Obersten Gerichtshof bestätigt worden war, obwohl es sich nach Auffassung des Blatts bei der Erteilung der Prokura nicht um einen bewilligungspflichtigen Dienstvertrag handelte. ${ }^{31}$ Auch Einbürgerungen wurden nun immer restriktiver gehandhabt: gemessen an den vielen österreichischen und reichsdeutschen Spezialarbeitern und Beamten, „die sich um die hiesige Staatsbürger-

26 So wurden hier etwa die Arbeitgeber angewiesen, Verzeichnisse der von ihnen beschäftigten Ausländer anzufertigen, eine Befugnis, die ausschließlich der Landesbehörde zukam. Vor dem 1. Mai 1923 zugereiste Ausländer genossen „vollständige Freizügigkeit“, mußten in solche Listen also nicht aufgenommen werden. Dissens bestand auch hinsichtlich der Bestimmungen über die Meldepflicht: Vgl. zu den Details MDHI 12 (1931), S. $738 \mathrm{f}$.

27 BayHStA, MA 103 108, Hilfsverein deutscher Reichsangehöriger zu Aussig an BayStMÄWA, 19. und 26. 3. bzw. 12. 4.1932. - BayStMÄWA an Pflock, 29.4.1932 bzw. an AA, undatiertes Konzept.

28 SÚA, MSP, Karton 1807, E 4/a, 1932, Gesandtschaft Prag an MZV, 9. 1. 1932.

29 BayHStA, MA 103 108, AA an BayStMÄWA, 25. 5. 1932.

30 DHI, Tätigkeitsbericht 1932, S. 40-42.

31 WI 14 (1932), S. 260. 
schaft bemühen, weil sie bei der herrschenden Arbeitslosigkeit nur schwer die Verlängerung der Aufenthaltsbewilligung erhalten und Gefahr laufen, ihre Existenz zu verlieren “, war die Naturalisierungsquote niedrig. ${ }^{32}$

Nun handelt es sich allerdings bei den geschilderten Beispielen durchwegs um solche aus dem Alltag der nachgeordneten Behörden. Auf der diplomatisch-politischen Ebene hingegen wirkte auch in dieser angespannten Situation die Macht des deutschen Reiches als eine Hemmschwelle. Ein wesentlicher Faktor war und blieb ja die Verwundbarkeit der ČSR angesichts der Tschechoslowaken im Reich: Die deutsche Volkszählung von 1925 hatte an die 223000 tschechoslowakische Staatsbürger in Deutschland erfaßt, etwa 202000 von diesen deutscher Muttersprache. 1930 stellten die Tschechoslowaken mit fast 63000 Beschäftigten - etwa 53000 davon in der Industrie - die mit Abstand größte Gruppe ausländischer Arbeitskräfte im Reich. ${ }^{33}$ Als ein Rundschreiben der Brünner Handelskammer im Dezember 1930 angesichts der grassierenden Arbeitslosigkeit in der Republik den „Mißstand“ der zahlreichen hochdotierten Ausländer brandmarkte, drohte die Prager deutsche Gesandtschaft mit der Mißgunst der öffentlichen Meinung in Deutschland; Retorsionen würden in diesem Fall auch die tschechoslowakischen Bürger deutscher Nationalität treffen. Als der Brünner „Tagesbote“ auf diese Einlassung hin eine massive Attacke gegen die Einmischung der Kammer ritt, wich Präsident Petr zurück. Zwar ließen, so Petr in einem Interview mit der „Prager Presse“, das mit Fug auch als eine Antwort des tschechoslowakischen Außenministeriums auf den reichsdeutschen Protest anzusehen war, manche Firmen die gebotene Rücksicht auf die Arbeitsmarktlage vermissen und beschäftigten Ausländer, wo qualifizierte Inländer zur Verfügung stünden; allerdings würden die Zahlen "gewöhnlich stark übertrieben“. Im Brünner Kammerbezirk handle es sich zudem durchwegs um „Spezialfachleute, deren Arbeit für die Hebung der heimischen Produktion nur förderlich ist“. Die Kammer beabsichtigte, sich ein genaues Bild zu verschaffen, „um jenen demagogischen Aktionen mit Erfolg entgegentreten zu können, die in der uninformierten Öffentlichkeit auch gegen die wirtschaftlich vorteilhaften Anstellungen von Ausländern von Tag zu Tag mehr an Boden gewinnen“. Das geltende Recht genüge "für die Wahrung unserer Interessen“ vollauf, ,irgendwelche Ausnahmebestimmungen“ seien, wenigstens für die nächste Zeit, nicht erforderlich. ${ }^{34}$

Zurückhaltung zeigte aber im allgemeinen auch die reichsdeutsche Seite: Munitioniert mit dem „Venkov" aber im allgemeinen vom 26. Februar 1931 und einigen anderen nationaltschechischen Pressestimmen, suchte Pflock Anfang April angesichts der sich verschlechternden Lage der Reichsdeutschen und der Bemäntelung nationalpolitischer Ressentiments mit ökonomischen Motiven ein weiteres Mal um die Unterstützung der Bayerischen Staatskanzlei an. Zwar lagen, wie Pflock zugab, „noch keine eklatanten Fälle" vor; mit Druck sei aber auch deswegen zu rechnen, weil die österreichisch-deutschen Zollunionspläne bei der deutschen Industrie der Republik eine so einhellig positive Aufnahme gefunden hätten. ${ }^{35}$ Als Ende des Monats die bayerische Regierung auf

32 Binder, Flucht.

33 MDHI 12 (1931), S. 289. - Wirtschaft und Statistik Nr. 19 (1928), S. 676. - BayHStA, MA 103 108, Reichsanstalt für Arbeitsvermittlung und Arbeitslosenversicherung an RAM, 7. 3. 1931.

34 ÖStA, BAA, Gesandtschaft Prag, o.S., Österreichische Gesandtschaft Prag an BAA, 7. und 13. 1. 1931. - Das Interview Petrs in der PP vom 10.1.1931.

35 Vgl. zur Frage der Zollunion oben, S. 187 ff.. 
Veranlassung Pflocks in Berlin einen Vorstoß für eine Registrierung aller Nationaltschechen im Reich unternahm ${ }^{36}$, blieb das Auswärtige Amt reserviert: Könne eine solche Aktion auch als "geeignete Warnung" dienen, so müsse doch jeder Anschein aggressiver Absichten zugunsten einer auf die guten Kontakte zu Beneš bauenden Politik „mit groBer Vorsicht" vermieden werden. Im übrigen riet das Auswärtige Amt im Einvernehmen mit der Reichsanstalt für Arbeitsvermittlung schon aus Kostengründen von einer solchen Sonderzählung $a b .^{37}$

Koch hatte, „zunächst ohne besondere Weisung“, bereits Anfang Januar 1931 Beneš sein Verständnis dafür bekundet, daß im Zeichen der Krise Genehmigungen verweigert und Ausländer an erster Stelle entlassen würden; angesichts der Nichtverlängerung „alter" Bewilligungen und dem willkürlichen Vorgehen nachgeordneter Behörden auf eigene Faust hatte er aber doch auch die Gefahr einer Abkühlung der Beziehungen in den Raum gestellt. Zwischen Beneš und dem deutschen Fürsorgeminister Czech bestand zu diesem Zeitpunkt bereits ein "Stillhalteabkommen" an der Arbeitsmarktfront; Beneš versicherte, Kontakt halten zu wollen, „falls eine Verschärfung der Verhältnisse eintreten sollte ${ }^{\text {“ }}{ }^{38}$ Das Fürsorgeministerium, Ende 1931 mit Pressevorwürfen wegen laxer Durchführung des Schutzgesetzes konfrontiert, rechtfertigte sich, ein schärferes Vorgehen sei angesichts der Auslandstschechoslowaken nicht möglich; Repatriierungen müßten aus Kostengründen und wegen der zusätzlichen Belastung des einheimischen Arbeitsmarktes unbedingt vermieden werden. ${ }^{39}$ "Neue Momente, die darauf schließen ließen, daß tschechoslowakischerseits für die nächste Zeit besondere gesetzliche Maßnahmen geplant würden, um ausländische und insbesondere Reichsangehörige von dem tschechoslowakischen Arbeitsmarkt grundsätzlich zu verdrängen “, waren Anfang Oktober 1932 nicht bekannt. Ebensowenig lagen neue Erkenntnisse über eine Verdrängung Reichsdeutscher im Einzelfall vor, so daß ein „begründeter Anlaß, in dieser Frage von neuem an die tschechoslowakische Regierung heranzutreten“, nicht gegeben war. ${ }^{40}$

36 BayHStA, MA 103 108, BayStMÄWA an AA, 24. 4. 1931. - BayHStA, MA 103 175, Endfassung dieses Schreibens, ohne relevante Änderungen.

37 BayHStA, MA 103 108, AA an BayStMÄWA, 6. 5.1931.

38 BayHStA, MA 103 108, Gesandtschaft Prag an AA vom 3.1.1931.

39 SÚA, MSP, Karton 1807, E 4/a/1931, Aktenvermerk MSP vom 5.12.1931.

40 BayHStA, MA 103 279, Bayerische Gesandtschaft Berlin an BayStMÄWA vom 7.10. 1932. 